\title{
Endoscopic ultrasound-guided fine-needle aspiration with on-site cytopathology versus core biopsy: a comparison of both techniques performed at the same endoscopic session
}

Authors

Institutions
Michael Lin ${ }^{1}$, Clark D. Hair ${ }^{2}$, Linda K. Green ${ }^{3}$, Stacie A. Vela ${ }^{4}$, Kalpesh K. Patel ${ }^{4}$, Waqar A. Qureshi ${ }^{5}$, Yasser H. Shaib ${ }^{5}$

Institutions are listed at the end of article. submitted

6. March 2014

accepted after revision

23. June 2014

\section{Bibliography}

DOI http://dx.doi.org/

10.1055/s-0034-1377611

Published online: 26.9.2014

Endoscopy International Open

2014; 02: E220-E223

(c) Georg Thieme Verlag KG

Stuttgart · New York

E-ISSN 2196-9736

\section{Corresponding author}

Yasser H. Shaib, MD

Michael E. DeBakey VA Medical Center

Division of Gastroenterology and Hepatology

2002 Holcombe Blvd

Houston

TX 77030

United States

Fax: +1-713-795-4471

yshaib@bcm.edu
Background: Endoscopic ultrasound (EUS)-guided fine needle aspiration (FNA) with bedside cytopathology is the gold standard for assessment of pancreatic, subepithelial, and other lesions in close proximity to the gastrointestinal tract, but it is time-consuming, has certain diagnostic limitations, and bedside cytopathology is not widely available.

Aims: The goal of this study is to compare the diagnostic yield of EUS-guided FNA with on-site cytopathology and EUS-guided core biopsy.

Methods: Twenty-six patients with gastrointestinal mass lesions requiring biopsy at a tertiary medical center were included in this retrospective analysis of a prospective cohort. Two core biopsies were taken using a 22 gauge needle followed by FNA guided by a bedside cytopathologist at the same endoscopic session. The diagnostic yield and test characteristics of EUS core biop-

\section{Introduction}

Endoscopic ultrasound (EUS)-guided fine needle aspiration (FNA) is a widely available, commonly used, and effective modality for the evaluation of various gastrointestinal and peri-intestinal masses including pancreatic, submucosal, and lymphatic lesions [1 -3]. Despite the diagnostic utility of EUS-guided FNA in the evaluation of mass lesions, there are several limitations to the procedure. The diagnostic yield of EUS-FNA is highly variable and influenced by the presence of an onsite cytopathologist. For example, EUS-FNA of pancreatic lesions has a diagnostic accuracy ranging from $78 \%$ to $95 \%$ [4], but these rates have been reported to be even lower for other targets $[5,6]$. Inadequate specimens are obtained in as many as $29 \%$ of patients who undergo EUS-FNA without immediate review by a bedside cytopathologist [7]. On-site cytopathological evaluation of FNA samples significantly decreases the number of inadequate samples as well as the number of needle sy and EUS FNA with bedside cytopathology were examined.

Results: The mean number of passes was 3.2 for FNA, and the mean procedure time was 39.4 minutes. The final diagnosis was malignant in $92.3 \%$. Sensitivity and specificity were $83 \%$ and $100 \%$, respectively, for FNA, and $91.7 \%$ and $100 \%$, respectively, for core biopsy. Diagnostic accuracy was $92.3 \%$ for FNA and $84.6 \%$ for core biopsy. The two approaches were in agreement in $88.4 \%$ with a kappa statistic of 0.66 ( $95 \%$ confidence interval $0.33-0.99$ ).

Conclusions: An approach using two passes with a core biopsy needle is comparable to the current gold standard of FNA with bedside cytopathology. The performance of two core biopsies is time-efficient and could represent a good alternative to FNA with bedside cytopathology.

passes needed $[8,9]$. Despite these advantages, many institutions lack immediate on-site interpretation by a cytopathologist during EUS-FNA. Another limitation of EUS-FNA is the inherent inadequacies of cytology itself. The absence of tissue architecture with FNA makes it difficult to diagnose stromal tumors and lymphomas $[10,11]$. More recently, studies have examined the utility of performing EUS-guided core biopsies as a way to overcome such limitations [12]. In the last decade, many studies have been published comparing the diagnostic yield of EUS-FNA (without onsite cytopathology) and EUS core needle biopsy for various gastrointestinal lesions. To our knowledge, there has not been a study comparing EUSguided core biopsy and EUS-FNA utilizing immediate bedside cytopathologist review. We hypothesize that the diagnostic yield of EUS core biopsy is comparable to the diagnostic yield of EUS-FNA combined with on-site cytopathology. 


\section{Methods}

\section{$\nabla$}

\section{Study design}

This study was conducted at a tertiary referral medical center. With the aim of increasing diagnostic yield, all patients who were referred for EUS-guided biopsy at the Michael E. DeBakey VA Medical Center (MEDVAMC) between October 2011 and January 2013 were considered for both FNA and core biopsy during the same endoscopic session. Core biopsy was not performed if patients had cystic lesions, small lesions (defined as $<1 \mathrm{~cm}$ ) legions with overlying vascular structures that precluded safe intervention, or if the advanced endoscopist performing the procedure felt the risks of multiple biopsies outweighed the benefits. Data for this study was prospectively collected as part of a database that includes all EUS procedures performed in our endoscopy unit. Informed consent was obtained from each patient. The protocol was approved by the Baylor College of Medicine IRB and the MEDVAMC Research and Development Committee.

\section{EUS-FNA and core biopsy}

In this study, back-to-back EUS-FNA and core biopsy were performed on all patients during the same endoscopic session. All procedures were performed by a single, experienced advanced endoscopist (YS). YS has performed over 1000 cases of EUS and EUS-FNA. A gastroenterology fellow partially assisted in some of these cases. The mass lesions were identified using endoscopic ultrasound and sampled via two core biopsies (Cook 22-gauge Pro-Core core biopsy needle, Cook Endoscopy Inc; Limerick, Ireland) followed by two FNA passes (Cook 22-gauge FNA needle, Cook Endoscopy Inc; Limerick, Ireland). Suction was used to enhance cell capture. A bedside attending cytopathologist was present during the procedure and evaluated all FNA samples. A minimum of two FNA passes were made and more passes were obtained as needed based on the bedside cytopathologist's assessment of the initial FNA samples. Core biopsies were processed and evaluated by a pathologist (LG) blinded to the FNA results. All FNA and core biopsy specimens felt to be malignant were reviewed and confirmed by a second pathologist. LG has over 25 years of experience as a cytopathologist. She is a consultant cytopathologist for the entire VA system. At the completion of the procedure, patients were monitored in the post-anesthesia care unit for adverse events. Adverse events occurring after the immediate post-procedure period were determined based on chart review.

\section{Outcomes}

The primary objective of this study was to determine the diagnostic accuracy of EUS-guided core biopsy when compared with the accuracy of the gold standard, EUS-FNA with a bedside cytopathologist. Accuracy was defined as the percentage of specimens in which the biopsy diagnosis was consistent with the final diagnosis (i.e. sum of true positives and true negatives). The final diagnosis was determined based on a combination of surgical pathology; biopsy of primary tumor or metastatic lesions; serial imaging including computed tomography (CT), magnetic resonance imaging (MRI), X-ray, ultrasound, and positron emission tomography (PET) imaging; labs such as tumor markers; and clinical course. For example, if pathological diagnosis could not be obtained after multiple attempts with various modalities, metastatic or rapidly growing lesions were considered to be malignant. Patients were monitored for at least 1 year following their EUS procedure. Secondary outcomes examined in this study in- cluded procedure time and adverse events. Procedure time was defined as the time from insertion of the endoscope to removal. Data for secondary outcomes were collected based on a review of anesthesia, post-anesthesia care unit, and electronic medical records.

\section{Statistical analysis}

The diagnostic accuracy, sensitivity, and specificity were calculated for both techniques. Diagnostic accuracy (defined as true positives and true negatives) was compared using the Chi-squared test. Agreement in diagnostic yield of EUS-guided core biopsy and EUS-FNA with bedside cytopathologist was assessed using the kappa statistic. A multivariate analysis was conducted to examine potential predictors of an accurate diagnosis. Data analysis was performed using JMP 7 software (SAS Institute, Cary, NC).

\section{Results}

During the period between October 2011 and January 2013, 45 patients were referred for EUS-guided biopsy at the MEDVAMC. Both interventions could not be performed on 19 of the 45 patients for the following reasons: seven cystic lesions, five lesions smaller than $1 \mathrm{~cm}$, four masses not seen during EUS, and three which were technically difficult to sample. Twenty-six patients were included in the final analysis. Nineteen $(73.1 \%)$ of the sampled lesions were pancreatic masses. Extrapancreatic lesions included peripancreatic lymph nodes, gastric lesions, para-aortic lymph nodes, mediastinal masses, and liver lesions. Patient demographics and other relevant clinical characteristics are provided in Table 1.

Results from the procedure are displayed in Table 2 . The mean number of passes was 3.2 for FNA. Mean procedure time was 39.4 minutes. There were no adverse events during or immediately following the procedure.

The final diagnosis was malignant in $92.3 \%$ of the cases and benign in $7.7 \%$ of the cases. Diagnostic accuracy was $84.6 \%$ (95\%Cl: $66.4-93.8 \%$ ) for EUS core biopsy and $92.3 \%$ (95\%CI: $75.5-97.8 \%$ ) for EUS-FNA. The difference in accuracy between the two approaches was not statistically significant $(P=0.14)$. The kappa statistic, which was calculated to measure the agreement in yield between EUS-FNA and EUS-guided core biopsy, was 0.62 (95\%CI 0.33 0.91 ). The sensitivity and specificity for EUS-FNA were $83 \%$ and $100 \%$, respectively. The sensitivity and specificity for EUS core biopsy were $91.7 \%$ and $100 \%$, respectively.

\section{Table 1 Patient characteristics.}

\begin{tabular}{|c|c|}
\hline \multicolumn{2}{|l|}{ Characteristics } \\
\hline Age, mean $\pm S D$, years & $66.8 \pm 8.9$ \\
\hline Sex, male, n (\%) & $25(96.2)$ \\
\hline Race, Non-Hispanic White, n (\%) & $16(61.5)$ \\
\hline \multicolumn{2}{|l|}{ Site of lesion } \\
\hline Pancreas, n (\%) & $19(73.1)$ \\
\hline Other, n (\%) & $7(26.9)$ \\
\hline
\end{tabular}


Table 2 Endoscopic ultrasound (EUS) characteristics.

\begin{tabular}{|c|c|}
\hline \multicolumn{2}{|l|}{ Diagnosis } \\
\hline Benign, n (\%) & $2(7.7)$ \\
\hline Malignant, n (\%) & $24(92.3)$ \\
\hline \multicolumn{2}{|l|}{ Diagnostic accuracy } \\
\hline FNA, n (\%) & $24(92.3)$ \\
\hline Core biopsy, n (\%) & $22(84.6)$ \\
\hline \multicolumn{2}{|c|}{ EUS-FNA test characteristics } \\
\hline Sensitivity, \% & 83 \\
\hline Specificity, \% & 100 \\
\hline \multicolumn{2}{|c|}{ EUS core biopsy test characteristics } \\
\hline Sensitivity, \% & 91.7 \\
\hline Specificity, \% & 100 \\
\hline \multicolumn{2}{|c|}{ Number of passes for FNA, $n$} \\
\hline Mean & 3.2 \\
\hline Range, min, max & 2,7 \\
\hline \multicolumn{2}{|l|}{ Procedure time, $\mathrm{min}$} \\
\hline Mean & 39.4 \\
\hline Range, min, $\max$ & 15,80 \\
\hline
\end{tabular}

EUS, endoscopic ultrasound; FNA, fine-needle aspiration.

\section{Discussion}

Endoscopic ultrasound-guided FNA with on-site cytopathology has become the gold standard in the evaluation of gastrointestinal and peri-intestinal mass lesions. This practice is not only costeffective [13] but immediate review of FNA by an on-site cytopathologist has been shown to increase diagnostic yield by as much as $18-26 \%$ [6, 7]. Unfortunately, this practice has not been universally embraced, most likely due to cost as well as lack of the necessary expertise and personnel. As such, there have been more and more studies published which examine alternatives to the on-site cytopathologist. One such alternative is the use of EUS-guided core biopsy [14]. Based on the results of our study, the diagnostic yield of two passes with a 22-gauge core biopsy needle is comparable to EUS-FNA with on-site cytopathology. The yield of EUS-FNA with a bedside cytopathologist in our study, irrespective of EUS-guided core biopsy, is similar to the yields published in other studies, which range from $78 \%$ to $89 \%$ [1517]. For institutions which cannot afford or do not have access to an on-site cytopathologist, performing two core biopsies may improve yields and prevent unnecessarily repeating the procedure. As far as we can tell, this is the only study which compares EUS core biopsy to our current gold standard of EUS-FNA in the same patients with on-site cytopathology review.

At this time, most of the published studies compare the diagnostic yield of EUS core biopsy using a 19-gauge needle and EUS-FNA without bedside cytopathology [16-19]. While diagnostic accuracy tends to vary depending on the site, there is generally no significant difference between the two modalities [17-19]. While the 19-gauge Trucut core biopsy needle appears to operate well at certain sites such as the esophagus and portions of the stomach, it was more difficult to use in the antrum/fundus of the stomach as well as the duodenal bulb [20]. Even with the new, European-designed 19-gauge fine needle biopsy device, which was designed to overcome the limitations of obtaining transduodenal samples from the pancreatic head, this process continues to be technically difficult [21]. As a result, other types of FNA and core biopsy needles are being developed and compared [22]. Bang et al. published a randomized trial which compared the diagnostic yield of the 22-gauge FNA needle and a new 22-gauge biopsy needle for EUS-guided sampling of solid pancreatic masses [23]. With the new 22-gauge biopsy needle, they were able to obtain transduodenal biopsies without difficulty, thus overcoming the limitations of the 19-gauge core biopsy needles. Interestingly, the new 22-gauge core biopsy needle was capable of obtaining cytology and histology specimens. Their study concluded that the diagnostic yield of the new 22-gauge biopsy needle is comparable to the 22-gauge FNA needle. The authors commented that the yield of histologic core tissue was unsatisfactory with the biopsy needle, but there was no statistically significant difference in the number of passes for diagnosis or number of cases where there was a failure to achieve the diagnosis between the two diagnostic modalities. Another study examined 62 patients with solid pancreatic lesions which were sampled by EUS-guided 22-gauge core biopsy needle and 25-gauge FNA needle at the same endoscopic session [24]. There was no difference in adequacy of the specimens obtained through FNA and core biopsy needles. Additionally there was a significant agreement between EUS-FNA and core biopsy ( $88.5 \%$ for positive agreement and 62.5 $\%$ for negative agreement).

In our study, EUS-guided core biopsies were obtained before EUS-FNA of the same lesion. As previously discussed, one of the inherent limitations of FNA is the lack of architecture which can be important in making certain diagnoses. This decision was made because we did not want the FNA to disrupt the underlying architecture and diminish the yield of core biopsy although this is only a theoretical risk. Additionally, we did not alternate the two modalities in an effort to keep more variables constant. It would be interesting to see whether results would be similar if EUS-FNA preceded the EUS-guided core biopsies.

There are several advantages to our study. It includes a wide spectrum of disease which is not limited to solid pancreatic mass lesions. It compares the yield of EUS-FNA and EUS core biopsy performed on the same lesion during a single endoscopic session. Studies which compare the yield of these two modalities generally use a criterion-standard reference method. While patients with suspected malignancies based on either of these diagnostic tests generally undergo a subsequent surgical resection whereby the final diagnosis may be confirmed, those with benign cytology or histology are followed clinically. The final diagnosis in these "benign" cases is generally determined after a certain period of time based on a patient's clinical course and/or subsequent studies which may include imaging or repeat endoscopy with or without sampling. The criterion-standard reference method is used in our study as well, but mass lesions were sampled simultaneously using EUS-FNA and core biopsies. In this manner, patients serve as their own control. Also, all core biopsy specimens were reviewed by a single pathologist who was blinded to the results of the preceding FNA specimens.

Despite performing EUS-guided core biopsies followed by multiple passes for FNA during the same endoscopic session, the procedure was performed safely and efficiently. There were no adverse events during or immediately following the procedure. Mean procedure time was 39.4 minutes. EUS-FNA has been accepted to be a safe intervention with a low post-procedural adverse events rate [25]. EUS-guided core biopsy (using the 19gauge Trucut needle) has also been shown to be safe, with an adverse events rate of approximately $2 \%$ [26]. Although more studies are needed, the EUS-guided core biopsy may eventually supplant EUS-FNA with on-site cytopathology as the gold standard. 


\section{Limitations}

Much like other published studies examining the diagnostic yield of EUS-FNA and EUS core biopsy, the primary limitation of this study is the small size of the study population. Additionally, we examined a heterogenous population of lesions in this study. Also, some patients referred for biopsy were not included because core biopsy could not be performed. As such, results are biased towards patients in whom core biopsy was technically feasible. While all EUS procedures were performed by a single, experienced endoscopist in our study which allows for standardization, diagnostic yield may vary at other institutions when endoscopy is performed by multiple physicians or less experienced physicians. This study was conducted at a single tertiary referral center, and as such, there was a disproportionate amount of malignant lesions. Also, a cost analysis was not performed in this study but it would be interesting to see whether EUS core biopsies are cost effective when compared with EUS-FNA + on-site cytopathologist. Lastly, our study was not designed or powered to show significant differences between EUS-FNA and core biopsies but to compare the accuracy of both approaches.

\section{Conclusion}

Based on our study, the diagnostic yield of two passes with a 22gauge core biopsy needle may be comparable to the current gold standard of FNA with a bedside cytopathologist when sampling gastrointestinal lesions. Large, prospective, randomized studies are still needed to further compare these two modalities. Eventually, an approach with two core biopsies could represent a time efficient and widely available alternative to FNA with a bedside cytopathologist.

Competing interests: There are no disclosures or conflict of interest for any of the authors.

Institutions

${ }^{1}$ Department of Medicine, Baylor College of Medicine, Houston, TX, United States

2 Department of Gastroenterology and Hepatology, Baylor College of Medicine, Houston, TX, United States

${ }^{3}$ Department of Pathology and Immunology, Michael E. DeBakey VA Medical Center, Baylor College of Medicine, Houston, TX, United States

${ }^{4}$ Department of Gastroenterology and Hepatology, Ben Taub General Hospital, Baylor College of Medicine, Houston, TX, United States

${ }^{5}$ Department of Gastroenterology and Hepatology, Michael E. DeBakey VA

Medical Center, Baylor College of Medicine, Houston, TX, United States

\section{References}

1 Vander Noot MR3rd, Eloubeidi MA, Chen VK et al. Diagnosis of gastrointestinal lesions by endoscopic ultrasound-guided fine-needle aspiration biopsy. Cancer 2004; 102: 157-163

2 Chen VK, Eloubeidi MA. Endoscopic ultrasound-guided fine-needle aspiration of intramural and extraintestinal mass lesions: diagnostic accuracy, complication assessment, and impact on management. Endoscopy 2005; 27: 984-989

3 Wiersema MJ, Vilmann P, Giovannini M et al. Endosonography-guided fine-needle aspiration biopsy: diagnostic accuracy and complication assessment. Gastroenterology 1997; 112: 1087-1095

4 Yoshinaga S, Suzuki H, Oda I et al. Role of endoscopic ultrasound-guided fine needle aspiration for diagnosis of solid pancreatic masses. Dig Endosc 2011; 23: 29-33
5 Kramer H, Sandes J, Post WJ et al. Analysis of cytological specimens from mediastinal lesions obtained by endoscopic ultrasound-guided fine-needle aspiration. Cancer 2006; 108: 206-211

6 Watson RR, Binmoeller KF, Hamerski CM et al. Yield and performance characteristics of endoscopic ultrasound-guided fine needle aspiration for diagnosing upper GI tract stroma tumors. Dig Dis Sci 2011; 56: $1757-1762$

7 Chang KJ, Wiersema M, Giovannini $M$ et al. Multi-center experience with endoscopic ultrasound guided fine needle aspiration of the pancreas. Gastrointest Endosc 1996; 43: S49 (abstract)

8 Iglesias-Garcia J, Dominguez-Munoz JE, Abdulkader I et al. Influence of on-site cytopathology evaluation on the diagnostic accuracy of endoscopic ultrasound-guided fine needle aspiration of solid pancreatic masses. Am J Gastroenterol 2011; 106: 1705 - 1710

9 Klapman JB, Logrono R, Dye CE et al. Clinical impact of on-site cytopathology interpretation of endoscopic ultrasound-guided fine needle aspiration. Am J Gastroenterol 2003; 98: 1289-1294

10 Jhala NC, Jhala DN, Chhieng DC et al. Endoscopic ultrasound-guided fine needle aspiration: a cytopathologist's perspective. Am J Clin Pathol 2003; 120: $351-367$

11 Ribeiro A, Vazquez-Sequeiros E, Wiersema LM et al. EUS-guided fineneedle aspiration combined with flow cytometry and immunocytochemistry in the diagnosis of lymphoma. Gastrointest Endosc 2001; 53: $485-491$

12 Saftoiu A, Vilmann P, Guldhammer Skov B et al. Endoscopic ultrasoundguided Trucut biopsy adds significant information to EUS-guided fineneedle aspiration in selected patients: a prospective study. Scand J Gastroenterol 2007; 42: 117-125

13 Nasuti JF, Gupta PK, Baloch ZW. Diagnostic value and cost-effectiveness of on-site evaluation of fine-needle aspiration specimens: review of 5,688 cases. Diagn Cytopathol 2002; 27: 1-4

14 Larghi A, Verna EC, Ricci R et al. EUS-guided fine-needle tissue acquisition by using a 19-gauge needle in a selected patient population: a prospective study. Gastrointest Endosc 2011; 74: 504-510

15 Chhieng $D C$, Jhala $D$, Jhala $N$ et al. Endoscopic ultrasound-guided fineneedle aspiration biopsy: a study of 103 cases. Cancer 2002; 96: 232 239

16 Wittmann J, Kocjan G, Sgouros SN et al. Endoscopic ultrasound-guided tissue sampling by combined fine needle aspiration and trucut needle biopsy: a prospective study. Cytopathology 2006; 17: 27-33

17 Varadarajulu S, Fraig M, Schmulewitz $N$ et al. Comparison of EUS-guided 19-gauge trucut needle biopsy with EUS-guided fine-needle aspiration. Endoscopy 2004; 36: 397-401

18 Storch I, Jorda M, Thurer R et al. Advantage of EUS trucut biopsy combined with fine-needle aspiration without immediate on-site cytopathologic examination. Gastrointest Endosc 2006; 64: 505-511

19 Storch I, Shah M, Thurer $R$ et al. Endoscopic ultrasound-guided fineneedle aspiration and trucut biopsy in thoracic lesions: when tissue is the issue. Surg Endosc 2008; 22: 86-90

20 Levy MJ, Wiersema MJ. EUS-guided trucut biopsy. Gastrointest Endosc 2005; 62: 417-426

21 Iglesias-Garcia J, Poley JW, Larghi A et al. Feasibility and yield of a new EUS histology needle: results from a multicenter, pooled, cohort study. Gastrointest Endosc 2011; 73: 1189-1196

22 Sakamoto H, Kitano M, Komaki $T$ et al. Prospective comparative study of the EUS guided 25-gauge FNA needle with the 19-gauge trucut needle and 22-gauge FNA needle in patients with solid pancreatic masses. J Gastroenterol Hepatol 2009; 24: 384-390

23 Bang JY, Herbert-Magee S, Trevino J et al. Randomized trial comparing the 22-gauge aspiration and 22-gauge biopsy needles for EUS-guided sampling of solid pancreatic mass lesions. Gastrointest Endosc 2012; 76: $321-327$

24 Berzosa M, Patel $K$, Vela $S$ et al. Comparison of a novel EUS core needle vs. standard FNA for pancreatic lesions: what's the verdict? [Abstract] Am J Gastroenterol 2012; 107: 109-S110

25 Al-Haddad M, Wallace MB, Woodward TA et al. The safety of fine-needle aspiration guided by endoscopic ultrasound: a prospective study. Endoscopy 2008; 40: 204-208

26 Thomas T, Kaye PV, Ragunath K et al. Efficacy, safety, and predictive factors for a positive yield of EUS-guided trucut biopsy: a large tertiary referral center experience. Am J Gastroenterol 2009; 104: 584-591 